\title{
Bioprocess intensification: aspirations and achievements
}

\section{Bill Whitford*, 1 iD}

${ }^{1}$ Cytiva, 100 Results Way, Marlborough, MA 01752, USA; *Author for correspondence: bill.whitford@cytiva.com

BioTechniques 69: 85-87 (August 2020) 10.2144/btn-2020-0072

First draft submitted: 19 May 2020; Accepted for publication: 26 May 2020; Published online: 25 June 2020

\section{KEYWORDS:}

biomanufacturing • biopharmaceutical $\bullet$ continuous $\bullet$ digital $\bullet$ intensification $\bullet$ modular $\bullet$ simplification $\bullet$ single-use

There are many drivers to intensify the manufacturing of a biological product. Advances are occurring throughout the biomanufacturing arena, from process development techniques to improved manufacturing platforms, equipment and facilities. Many employ the term 'bioprocess intensification' to refer to systems for producing more product per cell, time, volume, footprint or cost. This need is being driven by two emerging priorities: cost control and process efficiency. We are seeing great interest in the power of such disciplines as synthetic biology, process simplification, continuous bioprocessing and digital techniques in the optimization of bioprocess development and manufacturing. Other powerful disciplines here include process automation, improved monitoring and prefabricated facility modularity and podularity.

\section{Bioprocess intensification}

Defining bioprocess intensification (BI) is difficult, for at least four reasons. First, the number of new biological products, from exosomes to DNA vaccines, makes even cataloging new initiatives difficult. Second are the many biomanufacturing advances, including new process development techniques and new manufacturing platforms, equipment and facilities. Third, intensification efforts include many advances in science and technology as they support diverse analytic, diagnostic and therapeutic product applications [1].

The fourth difficulty relates to just what improvements in a process should be regarded as BI. Some have applied the term to most any process improvement, but many use it only when referring to getting more product from a process by producing more product per cell, time, volume, footprint or cost [2]. Used this way, BI refers to any activity that increases such common metrics as the cell-specific, volumetric or facility productivity. BI can be accomplished by such improvements as producing more product per time period upstream or retaining more quality product downstream. Defined this way, BI is a subordinate or supporting technology to the so-called nextgeneration or 'factory of the future' initiatives. BI can be enabled by such corollary technologies as continuous or digital biomanufacturing (Figure 1).

Many process improvements affect values and metrics not in the original purview of BI. For example, a development initiative intended to improve product quality can also reduce failed batches and thereby increase a plant's annual productivity. Limiting the categorization of an activity to its primary, intended goals can increase clarity. We herein define BI as an activity intended to increase productivity by any metric.

\section{Importance of B |}

$\mathrm{BI}$ is driven by many emerging trends. Cost control and process efficiency concerns are growing due to such initiatives as biosimilar licensing, 'in-county for-county' production and the market/product capabilities provided by single-use production systems and modular facilities [3].

The characteristic high labor and material costs of bioprocessing and a demand for extensive experimentation inspire process intensification. However, regulatory and product safety constraints have made biopharmaceutical production slower to incorporate efficiencies from newer manufacturing initiatives such as '4.0' digitalization, automation and operations integration.

Finally, there is often pressure to quickly develop a functional first-generation process, and only later turn attention to such goals as process capacity, robustness, consistency, efficiency, manufacturing flexibility and sustainability. Intensification of an established process helps accomplish this.

\section{Bl technologies \& techniques}

Biomanufacturing is in a remarkably dynamic era. The sheer range of new product types and applications, new manufacturing processes and support technologies and new facility designs and fabrication methods makes it impossible to provide a comprehensive view of the developments arising [4]. For this reason, upstream techniques are the main focus of this article. 


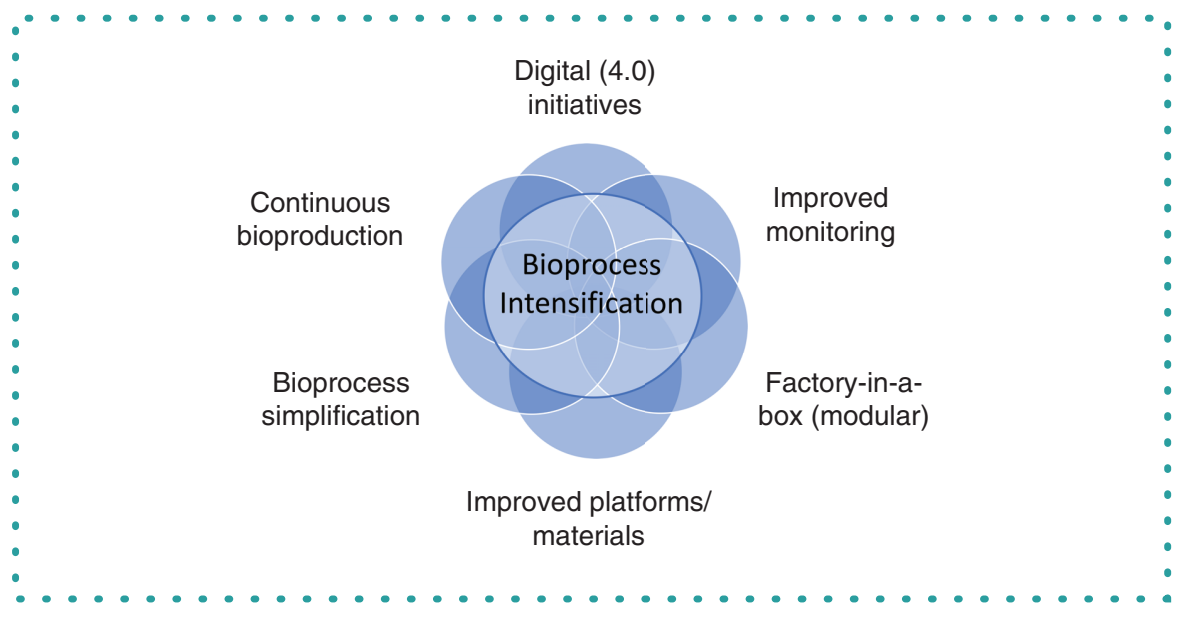

Figure 1. Recent technologies that can support bioprocess intensification..

Table 1. Operational states (modes) of both seed and production bioreactors establishing bioprocess intensification.

$\begin{array}{ll}\text { - Fed-batch culture } & \text { - Intensified perfused continuous } \\ \text { - Intensified seed train } & \text { - Perfused concentrated fed-batch } \\ \text { - High cell-count cryo stock } & \text { - Perfused high-productivity harvest } \\ \text { - Perfusion enabled continuous } & \text { - Repeated harvest/seeding iterations }\end{array}$

From an organizational level, modern enterprise asset management can provide better control over production, materials management, maintenance and repairs. Many data analysis approaches here can improve the performance of existing facilities while minimizing costs and risk.

Genetic/metabolic engineering of popular platforms can improve peak densities, culture duration and cell-specific productivity. While Chinese hamster ovary cells have become very popular in bioprocessing, other platforms such as human cell lines provide human-like post translational modifications. Escherichia coli is popular for some products due to its low cost and well-understood metabolism and genetics and its growth rate and productivity. Yeasts such as Pichea pastoris are gaining traction in some sectors [5]. Transgenic plants present such advantages as low cost, safety, ease of upscaling, stability and ability to produce N-glycosylated proteins [6].

Many examples in the seed train illustrate recently successful BI. Approaches include high-cell-density cryopreservation, supporting the elimination of early culture expansion cycles and thereby shortening its duration. Intensified perfusion in the seed train can also: support skipping an $\mathrm{n}-\mathrm{X}$ culture cycle, saving materials and time; allow seeding production reactors at higher densities, providing faster and/or higher final production reactor cell densities; and provide greater yields from a production reactor, as less nutrient complement is consumed in achieving peak densities. These activities are sometimes included in the concept of 'process simplification' [7].

While promoted for many other values, continuous bioprocesses can also provide intensification benefits by, for example, increasing the process productivity per time. A continuous operation can extend over many weeks or even months, eliminating process-slowing changeover iterations and culture expansion cycles. Intensified perfusion processes can dramatically increase the productivity in terms of both time and footprint (Table 1). Further optimizing a perfusion process involves modifying such parameters as media composition, operating cell density, perfusion rate and culture duration [8].

Batch culture medium or feed optimization can increase volumetric productivity; goals include establishing culture longevity over short-lived peaks and product secretion over culture mass. Optimal culture medium composition for the same platform employed in batch, fed-batch, perfusion-enhanced batch and perfusion-based continuous operations can vary greatly [9].

'Digital' approaches in biomanufacturing are showing promise to become major contributors to BI. Demonstrating the power of these '4.0' approaches in biopharma is how Moderna, a developer of mRNA-based therapeutics, won the 2019 ISPE Facility of the Future award. This highly automated enterprise delivered the first batch of COVID-19 vaccine to the National Institute of Allergy and Infectious Diseases 42 days from sequence identification [10].

BI data sources, transmission, storage and governance are maturing rapidly. Massive amounts of process data arise from new probes, sampling technologies, monitoring instrumentation, analytical procedure types and from high-throughput and multiplexed analytics [1].

Improvements in process control systems include supervisory control and data acquisition. Model-based adaptive systems can support closed-loop bioreactor control for many variables and actuate dynamic changes in output variables. Both bioreactor type and process control are now important factors to consider in BI [11].

'Digital twins' are virtual models of physical systems or processes that convert physical instrument operations to an in silico model [12]. They provide a digital transformation of the process supporting the analysis, optimization, prediction and even control of 
(A)

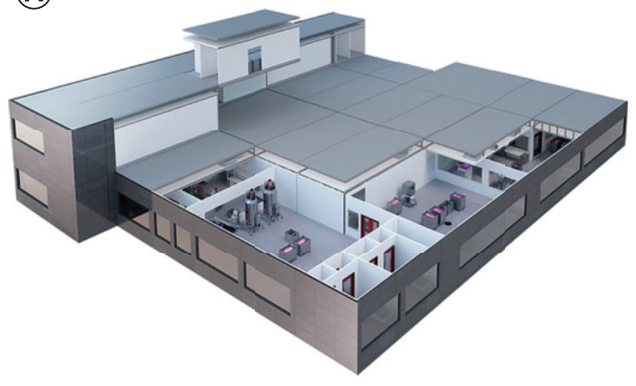

(B)

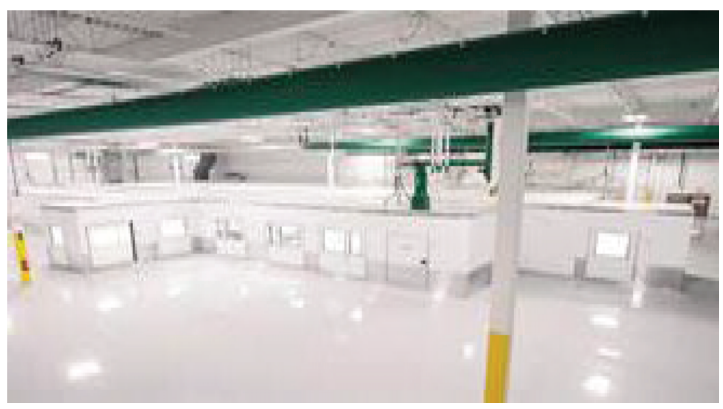

Figure 2. Modular biomanufacturing solutions enable intensification goals. (A) Cytiva KUBio ${ }^{\mathrm{TM}}$ modular mAb facility providing a FlexFactory ${ }^{\mathrm{TM}}$ single use production environment (www.cytivalifesciences.com). (B) Cleanroom POD ${ }^{\circledR}$ cluster by G-CON Manufacturing (www.gconbio.com).

a manufacturing processes. We envision mechanistic modeling and machine learning employed in digital twin process control to support a true 'factory of the future' [13].

BI power includes the industrial internet of things and newer data sources. So-called SMART techniques and automation in both process development and manufacturing process control will employ advanced monitoring and control techniques. Artificial intelligence will soon provide novel solutions to complex problems, even with less than optimally governed data; it will make appropriate decisions for bioprocess development, prediction, recommendations and control using advanced monitoring, big data processing capabilities and industrial connectivity [14].

Improvements in process monitoring include means to monitor entirely new process parameters and improvements in the monitoring of existing parameters. Improvements are occurring in basic sensing technologies, for example, electrochemical, capacitance, surface plasmon resonance, mass-based, optical, thermistor or piezoelectric-based. Smart sensors can convert surrogate measurements to a functional value in silico [15]. Automated sampling and distribution of culture samples is increasing in speed, precision and number of analytics supported [16].

Computer-aided biology divides activities into two realms: the digital and the physical. The digital, introduced above, includes advances from more powerful hardware to artificial intelligence. The physical includes systems supporting the automated incorporation of advanced experimental designs into actual laboratory experiments.

Developments in clone selection techniques include new criteria, such as culture behavior and robustness in the actual production media and mode and early use of a product quality profile. Cloning, cell-line development and gene editing are being advanced greatly through radical new technologies like microfluidics; one new approach directs millions of light-actuated pixels to move individual cells within a microfluidic chamber to be isolated, cultured, assayed and exported [17].

Single-use upstream processing results in faster product to market, smaller footprint, greater flexibility and reduced loss due to contamination. The most significant benefits in general are faster and easier tank preparation and turnaround times, lower capital and utility costs and reduced concern for cross-contamination [18].

Prefabricated and modular facilities, podular suites and modular operations units are perhaps the newest and most financially significant BI innovations (Figure 2); they can reduce footprint, capital expense, time to market, service needs and operating expenses. Facilities composed of prefabricated and modular units feature rapid build and deployment of cGMP-compliant design, materials, space allocation, workflow, equipment, utility, ventilation and climate control for both research and manufacturing. Prefabricated podular cleanrooms can be rapidly installed in unclassified (gray space) environments. Some prefabricated, modular, controlled-environment chambers combine principles of process intensification, automation and in-line chaining in self-contained ISO 14644-1 classified operating modules. They provide upstream, downstream and aliquoting capacity that drastically reduces facility footprint and capital and operational expenditures and are especially suited for such applications as viral vaccines, gene therapies and oncolytic viruses [19].

\section{Commercial availability of BI tools}

There is a difference between what exists in the university's or supplier's lab and what is commercially available to suppliers and manufacturers; however, there has been a steady progression of $\mathrm{BI}$ approaches from research to commercialized application. One example is new perfusion bioreactor, the Xcellerex APS; another is the launch of Fibro, a new rapid cycling fiber-based chromatography platform [20]. 


\section{Biomanufacturing sustainability}

Finally, there is growing concern over manufacturing-originated environmental stress. Interestingly, many process intensification initiatives designed to increase productivity also reduce the environmental footprint. For example, perfusion culture in the seed train can support the skipping of an entire culture expansion cycle, increasing cost-efficiency. This intensification not only reduces operational expenses, but also eliminates an entire culture cycle's worth of single-use materials, increasing the system's net sustainability.

\section{Financial \& competing interests disclosure}

The author is an employee of Cytiva. The author has no other relevant affiliations or financial involvement with any organization or entity with a financial interest in or financial conflict with the subject matter or materials discussed in the manuscript apart from those disclosed.

No writing assistance was utilized in the production of this manuscript.

\section{Open access}

This work is licensed under the Attribution-NonCommercial-NoDerivatives 4.0 Unported License. To view a copy of this license, visit http://creativecommons.org/licenses/by-nc-nd/4.0/

\section{References}

1. Current Developments in Biotechnology and Bioengineering: Bioprocess, Bioreactors and Controls. Larroche C, Sanroman MA, Du G, Pandey A (Eds). Elsevier (2016).

2. Whitford WG. Bioprocess Intensification: Technologies and Goals. In: Continuous Biomanufacturing: Process Control, Intensification and Digitalisation. Subramanian G (Ed.). (In Press).

3. Whitford WG. Single-use systems support continuous bioprocessing by perfusion culture. In: Continuous Processing in Pharmaceutical Manufacturing. Hoboken, NJ, USA 183-226 (2014).

4. Technology roadmapping. BioPhorum, London, UK (2017). http://www.biophorum.com/phorum/technologyroadmapping

5. Tripathi NK, Shrivastava A. Recent developments in bioprocessing of recombinant proteins: expression hosts and process development. Front. Bioeng. Biotechnol. 7, 420 (2019).

6. PR Newswire. Medicago's New Drug Submission accepted for scientific review by Health Canada: an important step for Medicago towards commercialization of its innovative influenza vaccine. http://www.prnewswire.com/news-releases/medicagos-new-drug-submission-accepted-for-scientific-review-by-health-canada-an-important-step-for-medicago-towards-co mmercialization-of-its-innovative-influenza-vaccine-300929093.html

7. Veyrat P. 5 awesome ideas for business process simplification (2016). http://www.heflo.com/blog/bpm/business-process-simplification-ideas

8. Godawat R, Konstantinov K, Rohani M, Warikoo V. End-to-end integrated fully continuous production of recombinant monoclonal antibodies. J. Biotechnol. 213, 13-19 (2015).

9. Whitford WG, Lundgren M, Fairbank A. Chapter 8: cell culture media in bioprocessing. In: Biopharmaceutical Processing. Jagschies G, Lindskog E, tącki K, Galliher P (Eds). Elsevier, Amsterdam, The Netherlands, 147-162 (2018).

10. Adams B. Moderna wins bragging rights as it kick-starts first experimental coronavirus clinical trial. (2020). http://www.fiercebiotech.com/biotech/moderna-wins-bragging-rights-as-itkickstarts-first-experimental-coronavirus-clinical-trial

11. R Pörtner O, Platas Barradas B, Frahm VC. Hass16 - Advanced process and control strategies for bioreactors. In: Current Developments in Biotechnology and Bioengineering Bioprocesses. Elsevier B.V., Amsterdam, The Netherlands, 463-493 (2017)

12. GE Digital. Digital Twins digitize assets and processes to enable better industrial outcomes. http://www.ge.com/digital/applications/digital-twin

13. Narayanan H, Luna MF, von Stosch M et al. Bioprocessing in the digital age: the role of process models. Biotechnol. J. 15(1), 1900172 (2020).

14. Mohammadi V, Minaei S. Artificial intelligence in the production process. In: Engineering Tools in the Beverage Industry. Woodhead Publishing, Cambridge, England, 27-63 (2019).

15. Wang B, Wang Z, Chen T, Zhao X. Development of novel bioreactor control systems based on smart sensors and actuators. Front. Bioeng. Biotechnol. 8, 7 (2020).

16. MAST platform: system for transferring samples from bioreactors to analytical devices. http://mastsampling.com/mast-platform/

17. Berkeley lights. https://www.berkeleylights.com

18. GE Healthcare. FlexFactory: gain efficiency and flexibility with a configurable single-use biomanufacturing platform. http://www.gelifesciences.com/en/us/solutions/bioprocessing/pro ducts-and-solutions/enterprise-solutions/flexfactory

19. Fibro chromatography. http://www.gelifesciences.com/en/us/solutions/bioprocessing/products-and-solutions/downstream-bioprocessing/fibro-chromatography?extcmp=--sem\&gclid =EAlaIQobChMIIr7n2P_v6AIVA56fCh2f7wLKEAAYASAAEgK9TvD_BwE

20. May M, GEN. Modular bioprocessing makes adaptability a snap. (2019). http://www.genengnews.com/insights/modular-bioprocessing-makes-adaptability-a-snap/ 